\title{
Does the Implementation of Children's Hospital in the Home affect Health Outcomes for Children with Chronic Respiratory Conditions? A non-contemporary retrospective cohort comparison study
}

\author{
Thomas Senyard ${ }^{1}$, Kelly Weir ${ }^{1}$, and Megan Rutherford ${ }^{1}$ \\ ${ }^{1}$ Gold Coast Hospital and Health Service
}

November 14, 2021

\begin{abstract}
Objective To investigate whether the implementation of a Children's Hospital in the Home (CHITH) service affects clinical and service outcomes for children with chronic respiratory conditions including cystic fibrosis and non-CF bronchiectasis. Study Design A non-contemporary retrospective cohort comparison study. Setting/Patients Children aged between 1 and 17.99 years who were admitted to Gold Coast University Hospital (GCUH) with a chronic respiratory condition for pulmonary optimisation were eligible. Methods A clinical audit was utilised to compare children with chronic respiratory conditions who were admitted to GCUH in (a) the 12 months prior to implementation of CHITH and (b) the 12-month period following the implementation of CHITH. Outcomes of interest included lung function; inpatient length of stay (days); duration on intravenous antibiotics and weight gain. Normally distributed data was compared using the t-test, while non-parametric data was analysed with the MannWhitney test. Results Data was analysed from 58 admissions, 27 (46.55\%) of which occurred in the 12 months of traditional management and $31(53.45 \%)$ in the 12 months following the implementation of the CHITH service. A statistically significant reduction in inpatient length of stay was noted following implementation of the CHITH service: 14.25 versus 6.0 days (pvalue $=0.0001$ ). The pre-CHITH cohort had a non-significant mean improvement of $7.625 \%$ in their FEV1 (pred\%) compared to $9.75 \%$ in the CHITH cohort ( $\mathrm{p}$-value $=0.44$ ). There was no significant difference in the secondary clinical outcomes. Conclusion The CHITH service provided equitable clinical outcomes for children with a chronic respiratory condition whilst significantly reducing inpatient length of stay.
\end{abstract}

Title: Does the Implementation of Children's Hospital in the Home affect Health Outcomes for Children with Chronic Respiratory Conditions? A non-contemporary retrospective cohort comparison study

Thomas Senyard B.Physio ${ }^{1-2}$, Megan Rutherford M.Physio², Kelly A Weir Ph.D. ${ }^{3-4}$

1. Adults and Children's Hospital in the Home, Gold Coast Hospital and Health Service, Gold Coast, Australia.

2. Physiotherapy Department, Gold Coast Hospital and Health Service, Gold Coast, Australia.

3. Allied Health Research, Gold Coast Hospital and Health Service, Gold Coast, Australia.

4. Menzies Health Institute Queensland, Griffith University, Gold Coast, Australia. ORCID: 0000-00025042-1925

Corresponding Author: Thomas Senyard

Address: Gold Coast University Hospital,

1 Hospital Boulevard, Southport, Queensland, Australia, 4215 
E-mail: thomas.senyard@health.qld.gov.au

Phone: +61756870038

Keywords: Hospital in the Home, Cystic Fibrosis, pulmonary optimisation,

Objective

To investigate whether the implementation of a Children's Hospital in the Home (CHITH) service affects clinical and service outcomes for children with chronic respiratory conditions including cystic fibrosis and non-CF bronchiectasis.

\section{Study Design}

A non-contemporary retrospective cohort comparison study.

\section{Setting/Patients}

Children aged between 1 and 17.99 years who were admitted to Gold Coast University Hospital (GCUH) with a chronic respiratory condition for pulmonary optimisation were eligible.

\section{Methods}

A clinical audit was utilised to compare children with chronic respiratory conditions who were admitted to GCUH in (a) the 12 months prior to implementation of CHITH and (b) the 12-month period following the implementation of CHITH. Outcomes of interest included lung function; inpatient length of stay (days); duration on intravenous antibiotics and weight gain. Normally distributed data was compared using the t-test, while non-parametric data was analysed with the Mann-Whitney test.

\section{Results}

Data was analysed from 58 admissions, $27(46.55 \%)$ of which occurred in the 12 months of traditional management and $31(53.45 \%)$ in the 12 months following the implementation of the CHITH service. A statistically significant reduction in inpatient length of stay was noted following implementation of the CHITH service: 14.25 versus 6.0 days (p-value $=0.0001$ ). The pre-CHITH cohort had a non-significant mean improvement of $7.625 \%$ in their FEV1 (pred\%) compared to $9.75 \%$ in the CHITH cohort ( $\mathrm{p}$-value $=0.44$ ). There was no significant difference in the secondary clinical outcomes.

\section{Conclusion}

The CHITH service provided equitable clinical outcomes for children with a chronic respiratory condition whilst significantly reducing inpatient length of stay.

\section{INTRODUCTION}

Hospital in the home (HITH) programs have been in place within Australia since their introduction in the state of Victoria in 1994[1]. The aim of HITH is to provide hospital equivalent care within the home environment. Proposed benefits to the healthcare system include alleviating pressure on inpatient beds, reduced health care costs and improved flexibility of health care services. Benefits to patients include reduced risk of cross infection, reduced interruption to education and career, reduced periods as an inpatient and improved quality of life[1-3].

The Gold Coast Hospital and Health Service (GCHHS) implemented a Children's Hospital in the Home (CHITH) service at the end of December 2017. The team consisted of nursing and physiotherapy with medical oversight provided by hospital paediatricians. The CHITH service most commonly provides intravenous antibiotics (IVABs), wound management, basic orthopaedic/neurological rehabilitation and other services that would have previously required an inpatient admission. Common admissions to CHITH are children with a chronic respiratory condition such as cystic fibrosis $(\mathrm{CF})$, non-CF bronchiectasis or pulmonary ciliary dyskinesia (PCD). These children routinely require admissions to hospital for a 14-day pulmonary optimisation. These optimisations involve the provision of IVABs, medical reviews, intensive airway clearance 
and nutritional supplementation[4]. This is costly to the health services, utilises inpatient beds, is costly and arduous for families, and impact on children's education and social participation [5-7]. As a result, a home-based service providing optimal support for children, whilst reducing inpatient stay, has the potential to provide many benefits.

Previous studies have investigated the efficacy of home-based delivery of care for patients with CF[7-18]. However, study designs and service models have varied widely resulting in inconsistent outcomes. Despite this variance, Bryant \& Katz [7] found, in their systematic review of antibiotic therapy at home for acute infections, that there was no significant difference in children's lung function between hospital and homebased services. They also found that management within the home was cheaper with increased patient satisfaction. Chrysochoou et al[13] found in a study of 35 children that the provision of at-home treatment with IVAB therapy had equitable clinical outcomes such as lung function (forced expiratory volume in once second, FEV1) and weight gain, compared to children treated in hospital, with substantial improvements in quality of life and economic savings. Collaco et al[10], likewise found equivalent outcomes between home and hospital based IV antibiotic therapy in terms of long-term maintenance of FEV1, change in lung function and time to next admission in their cohort of children and adults. Conversely, Lavie et al[12], who studied 54 adult and paediatric patients (age range 12-47 years), found greater improvements in FEV1, shorter duration of treatment and a trend towards greater weight gain in hospital treated patients. Thornton et al[11] found better respiratory outcomes and weight gain in a retrospective study of 116 adult patients treated in hospital compared to home. These differences may have been due to reduced medical input/oversight in the home environment, reduced access to services normally provided intensely as an inpatient such as physiotherapy and dietetics, difference in compliance/timing of medications, lack of rest and variations in the monitoring of patients[3]. Due to this disparity in results and HITH models of care the Cystic Fibrosis Foundation recommends that pulmonary exacerbations are only managed at home if there are equivalent support and resources to the hospital setting[19].

There has been very limited research into the management of non-CF bronchiectasis within the home in children. However, a recent study identified the significant burden that this disease places on patients, their families and the health system. Issues of note include increased absenteeism from school, carer's leave and high levels of antibiotic use when treated in hospital[20]. Two other recent studies found that children with non-CF bronchiectasis could be safely managed within the home environment and achieve comparable outcomes while reducing healthcare costs[21, 22].

The aim of this study was to investigate the impact of introducing a CHITH service for children in an Australian regional hospital, including nursing and physiotherapy care, on patients' clinical outcomes compared to the preceding 12 months, under a more traditional inpatient model.

\section{METHODS}

\section{Ethical approval:}

This study was approved by the Gold Coast Hospital and Health Service (GCHHS) Human Research Ethics Committee (LNR/2019/QGC/52722).

\section{Study Design:}

A non-contemporary retrospective cohort comparison study was utilised to compare two groups: (a) a pre-CHITH "traditional" model of care were children with chronic respiratory conditions treated in the 12 months prior to the implementation of CHITH (January 2017-December 2017) and (b) "CHITH" were children admitted in the 12-month period following the implementation of CHITH (January 2018-December 2018).

\section{Study Population:}

The study population were children aged between 1 and 17.99 years who were managed under the Gold Coast University Hospital (GCUH) paediatric department for pulmonary optimisation with a chronic respiratory 
condition. The chronic respiratory conditions included were CF, non-CF bronchiectasis and PCD. Patients were excluded if they were within the age range but were managed by the adult respiratory team, did not have a pulmonary optimisation during their admission, were admitted to ICU or were on the waiting list for a lung transplant.

\section{Care Pathway:}

The pre-CHITH group were treated with the traditional model of inpatient paediatric care at GCUH. This included 24 hour medical and nursing care with extensive allied health input for approximately 14 days. The CHITH group were managed initially utilising the same traditional model of care with the option of Children's Hospital in the Home if it was clinically and socially safe to do so. The decision regarding patient suitability for CHITH was made by the treating medical team, in discussion with the patient and their family. As a result, many patients were managed under both the inpatient and CHITH model during the same admission. The care provided under the CHITH service included daily nursing for provision of intravenous antibiotics, management of dressings and observations. Physiotherapy was provided twice a day during the week and once a day over weekends to reflect a similar service provided during an inpatient admission. Medical oversight was provided by the treating team with input from the paediatric respiratory nurse practitioner and the CHITH consultant physician.

\section{Outcome Measures:}

- Inpatient length of stay (days): This was measured from the day of inpatient admission to hospital to the day of discharge or to the day they began being managed at home. (hospital stay)

- Length of time (days) on IV antibiotics

- Lung Function: Pulmonary function testing including forced expiratory volume in 1 second (FEV1), forced vital capacity (FVC) and Forced Expiratory Flow averaged over the middle portion of FVC (FEF25-75\%) were utilised as the primary outcomes to compare cohort (a) and cohort (b). FEV1, in particular, is a well-established outcome measure for disease progression in chronic respiratory disease[23, 24].

- Weight: Weight was measured on A\&D UC-321 scales by nursing staff and recorded to the nearest gram (g) at admission and discharge from GCHHS service. Nutritional status is particularly important as previous studies have indicated that it correlates with poorer outcomes, particularly if lean body mass is affected[25]. Weight for age z-scores were utilised to reduce errors that may occur due to differences in age group.

- Length of time (days) receiving the CHITH service: This outcome measure was included as the CHITH service provided physiotherapy follow-up, when clinically indicated, after the cessation of IVABs that extended the length of under the CHITH team. Our intention was not to confuse this with the time on IV antibiotics.

\section{Data Analysis}

Demographic data was described using number, percentages, mean and standard deviations (SD) or median and interquartile range. All outcome data was tested for normality using the Shapiro-Wilk test to assess the distribution of the data. Data analysis was carried out using Stata 15.1 software. If data was normally distributed an independent samples T-test was utilized to compare groups. Non-normally distributed data was analysed using the Mann-Whitney U test.

\section{RESULTS}

There were 89 admissions of 39 patients with a chronic respiratory condition between the ages of 1-17.99 to GCUH during the study period. Of these, $79(89 \%)$ were admitted for a pulmonary optimisation. Fifty-eight of these admissions were for CF, 11 were for non-CF bronchiectasis and 10 for PCD. When these admissions were reviewed it was noted that there was a significant difference in demographics and numbers for the pre-CHITH and CHITH cohorts for non-CF bronchiectasis and PCD (Table 1). As a result, it was decided that the data for cystic fibrosis would be analysed separately and fata for the other respiratory conditions 
would be described for completion, but excluded from further analysis.

Data was analysed from 58 pulmonary optimisation admissions of 27 individual patients during the two-year study period. Of these, $27(46.55 \%)$ admissions occurred in the year prior to the implementation of CHITH and $31(53.45 \%)$ occurred in the year following the implementation of CHITH. It should be noted that of the 31 patients with CF who were admitted for pulmonary optimisation in the 12 months that CHITH was implemented, 27 (87.1\%) were managed under the CHITH model at some stage during their admission with a mean period on CHITH of 11.4 days.

In the 2-year study period there were $37(63.8 \%)$ male admissions compared to $21(36.2 \%)$ females, both cohorts had similar gender ratios. There was no significant difference found in the age of admission for the pre-CHITH cohort (mean 11.45, range 2.36-16.7 years) and the CHITH cohort (mean 11.70, range 2.5-16.84 years $(\mathrm{p}=0.53)$.

\section{Service Duration}

The average length of stay was found to be 14 days in the pre-CHITH cohort compared to the 16 days in the CHITH cohort, which was found to be a significant difference between the two groups $(p=0.013)$. Average duration of time on IVABs was found to be 14 days in the both Pre-CHITH and CHITH cohorts ( $\mathrm{p}=0.49)$. Average hospital length of stay in the pre-CHITH cohort of 14.25 days was significantly longer than the CHITH cohort who had an average of 6 days $(\mathrm{p}=0.0001)$.

\section{Lung Function}

The primary outcome measures utilised were FEV1, FVC and FEF25-75\%. When data was missing due to patients not completing a lung function test on either admission or discharge, these patients were excluded. Data was collected from 24 lung function tests from the pre-CHITH cohort and 25 from the CHITH cohort.

FEV1 (Pred\%) on admission was $75.04 \%$ in the pre-CHITH cohort was similar to $71.2 \%$ in the CHITH group $(\mathrm{p}=0.51)$. Average improvement in FEV1 (Pred\%) for the pre-CHITH cohort at $7.625 \%$ was again similar to the CHITH cohort at $9.75 \%(\mathrm{p}=0.44)$.

There was no significant difference found on admission between the pre-CHITH group and CHITH regarding FVC (Pred\%) with the mean being $87.25 \%$ and $81.96 \%$ respectively. The average change in FVC (Pred\%) for the pre-CHITH cohort was $6.21 \%$ compared to $10.3 \%$ in the CHITH cohort $(\mathrm{p}=0.19)$.

On admission the pre-CHITH cohort achieved an average FEF25-75\% (Pred\%) of 53.5\% with the CHITH cohort achieving $54.16 \%(\mathrm{p}=0.27)$. Again there was no difference in average FEF $25-75 \%$ improvement between groups, which was $9.21 \%$ in the pre-CHITH cohort compared to $7.67 \%$ in the CHITH cohort $(\mathrm{p}=0.965)$.

\section{Weight}

Similarly to lung function, patients who had missing data for weight were excluded from analysis for this component. Data was collected from 26 patients in the pre-CHITH cohort and 30 from the CHITH cohort. Weights were collected at time of admission to the hospital service and on the day of cessation of IVABs. On admission, the average weight of patients was 43.04 kilograms in the pre-CHITH cohort and 44.68 kilograms in CHITH cohort, with no significant differences between groups $(\mathrm{p}=0.73)$. Likewise, weight for age $\mathrm{z}$-scores were not found to be significantly different $(\mathrm{p}=0.12)$ with a median of 0.265 and 0.645 respectively. Each cohort had an increase in weight from admission to discharge, with the pre-CHITH cohort improving by $0.99 \mathrm{~kg}$ on average and the CHITH cohort improving by $0.73 \mathrm{~kg}(\mathrm{p}=0.48)$.

\section{DISCUSSION}

This study reviewed whether the implementation of a paediatric hospital in the home service in an Australian regional health service could achieve equitable clinical outcomes for children with a chronic respiratory condition whilst decreasing hospital stay. We found that the CHITH model of care provided similar clinical outcomes for children with CF whilst decreasing hospital length of stay. However, due to limited numbers 
of patients with non-CF bronchiectasis and PCD during the study period we could not make any firm conclusions about the benefit of the model of care regarding this cohort. Although a recent study by O'Rourke et al[22] indicates that children with non-CF bronchiectasis can be managed safely at home while achieving comparable outcomes.

Previous studies have found that patients managed at home have an increased length of time on IVABs compared those managed within a hospital[7, 12,16], however our study found that the median time on IVABs was 14 days for both cohorts. This may have been due to our hospital's adherence to best practice guidelines $[4,19]$ which state that the "routine" provision of IVABs for pulmonary exacerbations is 14 days. However, the same guidelines recommend that the duration of treatment should be based on clinical judgement and, therefore, it would be expected that with adequate medical oversight included in this study this should not have been an issue. This study found that the introduction of the CHITH program significantly reduced the patient's inpatient length of stay from a median of 14 to 6 days. It can also be noted that $25 \%$ of patients managed in the CHITH cohort had an inpatient stay of less than 2 days, representing a substantial reduction in inpatient bed days compared to traditional care[4]. This may be offset by the additional employment of nursing and physiotherapy staff with related expenditure on travel to patients' homes. Further investigation of the health economics or cost-benefits between the two models would be essential to fully analyse the CHITH model. Previous studies have shown that home-based management reduces costs[11], but this was with reduced support at home, and not reflective of our model. A recently published study by Goyal et al[21] of the costs of hospital admission for exacerbation of bronchiectasis reported an average saving of $\$ 7766.19$ (Australian dollars) (approximately $\$ 5532$ USD) per admission when CHITH was utilised at Queensland Children's Hospital (QCH). The CHITH model of care utilised in our study is based closely on the QCH model and likely reflects similar savings in our service.

Pulmonary function, and in particular FEV1, is generally considered to be one of the most important indicators of disease progression[4, 23]. This study found no significant difference in FEV1 between patients treated within the traditional model and those who were managed following the implementation of CHITH. As discussed earlier, there is conflicting evidence in the research regarding lung function and home-based management. Our study provided clinical oversight and therapy, particularly through daily nurse administration of IVABs and twice-daily physiotherapy, similar to that provided during an inpatient admission. This closely aligns with the recommendations of the Cystic Fibrosis Foundations whereby the provision of resources and support to home based care should be similar to the hospital setting[19].

Children in the CHITH cohort gained weight during their admission, however the mean change was 260 grams less than the traditional model. Weight for age z-scores reflected this with a non-significant difference in change between the two cohorts. It should be noted that the median z-score of the CHITH cohort on admission was greater compared to the traditional model ( 0.645 versus 0.265 respectively), which may represent a better nutritional status in the CHITH cohort on admission resulting in a lower capacity for weight gain compared to the traditional cohort. Access to close nutritional monitoring by a dietitian was limited to the period that participants were within the hospital setting. As a result of shorter hospital length of stay in the CHITH model, this would have been restricted and may have impacted on weight gain. Other potential explanations include children in hospital (traditional model) having less opportunity for physical exercise in hospital, as they are confined to isolation rooms to reduce risk of respiratory infections resulting in less energy expenditure. Additional, family meals at home may have greater variability in nutritional content compared to dietitian prescribed meals and supplements when the child is in hospital. These findings reflect previous studies of paediatric and mixed-age populations that found no significant difference in weight gain during hospital or home based IV therapy $[8,9,17,18]$. Two studies of adults with CF found that hospital patients gained significantly more weight than those treated at home[11, 12]. Both studies identified that the patients managed at home were not provided with a similar level of support to those based within the hospital.

One reason for the difference in findings compared to previous studies may be the attempt to provide a more intensive, "hospital-like" service within the home. Patients are reviewed by a nurse daily, physiotherapists 
visit twice per day on weekdays and once per day on weekends. Paediatric consultants and respiratory nurse practitioners are easily contacted via phone and an outpatient review arranged if there are concerns. Sequeiros and Jarad[3] in particular expressed the concerns of many health professionals that patients on HITH frequently have reduced medical input, reduced physiotherapy and dietetics management, potential lack of compliance with IVABs if poorly monitored, lack of rest and a reliance on patients to recognise and react to complications. Whilst these are fair concerns, by providing a more supportive environment this study highlights that implementing a CHITH program can be done without significantly impacting patients' clinical outcomes.

The strengths of this study are that it offers an analysis of a pragmatic, real-life practice change from a traditional inpatient model of care to a CHITH model, and the impact of this implementation on patient outcomes and hospital LOS. This study analysed the whole cohort of patients rather than patients being allocated to an inpatient model care or a HITH service. This was necessary, as it was a retrospective review of the service but also eliminated some of the selection bias that previous reviews[7] have identified; primarily, that "sicker" patients are placed in the hospital group and "healthier" patients managed at home. This study allowed treating clinicians to clinically reason and coordinate with the family regarding the location their child should be treated based on physical, mental and social factors. As discussed in the results, this allowed 27 of 31 patients to be managed at home for an average of 11.4 days with equitable clinical outcomes.

Due to the retrospective nature of this study, there are limitations that should be considered. This study was heavily reliant on the accurate testing and assessment of clinicians as part of routine clinical practice. As is common for retrospective studies, this means that there was missing data for patients in both lung function and weight measure, as happens in clinical practice. It should also be noted that some patients had multiple admissions during the study period, which may have skewed our data. This was particularly true for the bronchiectasis and PCD data, but less so for CF. We reported patient data on each admission, rather than each admission representing a separate child. Future studies could report on single admission data (one admission per child) and potentially controlled trials would address this. To be adequately powered we would require much higher patient numbers which would take many years to obtain in our hospital's CHITH service. It may however, be achieved in a shorter time frame through a prospective multisite trial. As this is a non-contemporaneous study there was the potential for cohorts to be exposed to different variables, conditions and medical advances. This was particularly pertinent for the cystic fibrosis population as the first cystic fibrosis transmembrane conductance regulator (CFTR) protein modulating medication (Orkambi) became available on the pharmaceutical benefit scheme in Australia in October of 2018. CFTR modulating medications treat the underlying cause of cystic fibrosis and may have had an impact on outcomes, however many patients would not have had the opportunity to start these medications during the study period.

\section{CONCLUSION}

Our study found that the implementation of a well-resourced CHITH service can provide equitable clinical outcomes for children with CF whilst significantly reducing the need for a prolonged hospital-based admission. Further prospective studies with larger numbers of children (including non-CF bronchiectasis and PCD), longer study duration and long-term patient follow-up should be considered. Prospective implementation studies and health economic evaluations would provide further evidence for cost effectiveness of the CHITH model of care, as would qualitative or mixed method studies on patient acceptability, quality of life and impact.

\section{Declaration of interest:}

The authors report no conflicts of interest. The authors alone are responsible for the content and writing of this paper.

\section{Acknowledgements:}

We would like to acknowledge the GCHHS Allied Health Research Clinical Backfill Scheme for a small grant to support clinical backfill for TS to undertake research activities. 


\section{References}

1. Montalto, M. The 500-bed hospital that isn't there: the Victorian Department of Health review of the Hospital in the Home program. Med J Aust. 2010; 193 (10): 599-601.

2. Balaguer, A. and Gonzalez de Dios, J. Home versus hospital intravenous antibiotic therapy for cystic fibrosis. Cochrane Database Syst Rev. 2015; 12: p. CD001917.

3. Sequeiros, I. and Jarad, N. Home intravenous antibiotic treatment for acute pulmonary exacerbations in cystic fibrosis - Is it good for the patient? Ann Thorac Med. 2009; 4(3): 111-4.

4. Castellani, C., Duff, A., Bell, S., Heijerman, H., Munck, A., Ratjen, F., Sermet-Gaudelus, I., Southern, K., Barben, J., Flume, P., et al. ECFS best practice guidelines: the 2018 revision. J Cyst Fibros. 2018; 17(2): 153-178.

5. Champaloux, S., and Young, D. Childhood chronic health conditions and educational attainment: a social ecological approach. J Adolesc Health. 2015; 56(1): 98-105.

6. Compas, B., Jaser, S., Dunn, M., and Rodriguez, E. Coping with chronic illness in childhood and adolescence. Annu Rev Clin Psychol. 2012; 8: 455-80.

7. Bryant, P. and Katz, N. Inpatient versus outpatient parenteral antibiotic therapy at home for acute infections in children: a systematic review. Lancet Infect Dis. 2018; 18(2): e45-e54.

8. Termoz, A., Touzet, S., Bourdy, S., Decullier, E., Bouveret, L., Colin, C., Nove-Josserand, R., Reix, P., Cracowski, C., Pin, I. et al. Effectiveness of home treatment for patients with cystic fibrosis: the intravenous administration of antibiotics to treat respiratory infections. Pediatr Pulmonol. 2008; 43(9): 908-15.

9. Chrysochoou, E., Hatziagorou, E., Kirvassilis, F., and Tsanaka, J. Home intravenous antibiotic therapy in children with cystic fibrosis: clinical outcome, quality of life and economic benefit. Hippokratia. 2016; 20 (4): $279-83$

10. Collaco, J., Green, D., Cutting, G., Naughton, K., and Mogayzel, P. Location and duration of treatment of cystic fibrosis respiratory exacerbations do not affect outcomes. Am J Respir Crit Care Med. 2010; 182(9): 1137-43.

11. Thornton, J., Elliot, R., Tully, M., and Webb, K. Long term clinical outcome of home and hospital intravenous antibiotic treatment in adults with cystic fibrosis. Thorax. 2004; 59(3): 242-6.

12. Lavie, M., Vilozni, D., Sokol, G., Somech, R., Szeinberg, A., and Efrati, O. Hospital versu home treatment of respiratory exacerbations in cystic fibrosis. Med Sci Monit. 2011; 17 (12): 698-703.

13. Hatziagorou, E., Chrysochoou, E., Kirvassilis, F., and Tsanakas, J. 270 Home antibiotic treatment in cystic fibrosis: An effective, cost saving and preferred choice among patients with CF. J of Cyst Fibros; $2015 ; 14$.

14. Moran, F., Barr, C., Kerr, K., and Tannenbaum, E. A review of hospital and hospital in the home (HiTH) service at a paediatric cystic fibrosis (CF) centre. J Cyst Fibros. 2014; 13: S103.

15. Wolter, J., Bowler, S., Nolan, P., and McCormack, J. Home intravenous therapy in cystic fibrosis: a prospective randomized trial examining clinical, quality of life and cost aspects. Eur Respir J. 1997; 10 (4): 896-900.

16. Nazer, D., Abdulhamid, I., Thomas, R., and Pendleton, S. Home versus hospital intravenous antibiotic therapy for acute pulmonary exacerbations in children with cystic fibrosis. Pediatr Pulmonol. 2006; 41(8): 744-9.

17. Riethmueller, J., Busch, A., Damm, V., Ziebach, R., and Stern. Home and hospital antibiotic treatment prove similarly effective in cystic fibrosis. Infection. 2002; 30(6): 387-91. 
18. Proesmans, M., Heyns, L., Moons, P., Havermans, T., and De Boeck, K. Real life evaluation of intravenous antibiotic treatment in a paediatric cystic fibrosis centre: outcome of home therapy is not inferior. Respir Med. 2009; 103(2): 244-50.

19. Flume, P., Mogayzel, P., Robinson, K., Goss, C., Rosenblatt, R., Kuhn, R., Marshall, B., and the Clinical Practice Guidelines for Pulmonary Therapies Committee. Cystic Fibrosis Pulmonary Guidelines. Am J Respir Crit Care Med. 2009; 180(9): 802-808.

20. Lovie-Toon, Y.G., Grimwood, K., Byrnes, C., Goyal, V., Busch, G., Masters, B., Marchant, J., Buntain, H., O'Grady, K., and Chang, A. Health-resource use and quality of life in children with bronchiectasis: a multi-center pilot cohort study. BMC Health Serv Res. 2019; 19(1): 561.

21. Goyal, V., McPhail, S., Hurley, F., Grimwood, K., Marchant, J., Masters, B., and Chang, A. Cost of hospitalization for bronchiectasis exacerbation in children. Respirology. 2020; 25: 1250-1256.

22. O'Rourke, C., Schilling, S., Martin, R., Joyce, P., Chang, A., and Kapur, N. Is out-patient based treatment of bronchiectasis exacerbations in children comparable to inpatient based treatment? Pediatr Pulmonol. 2020; 55(4): 994-999.

23. Szczesniak, R., Heltshe, S., Stanojevic, S., and Mayer-Hamblett, N. Use of FEV1 in cystic fibrosis epidemiologic studies and clinical trials: A statistical perspective for the clinical researcher. J Cyst Fibros. 2017; 16(3): 318-326.

24. Stanojevic, S. and F. Ratjen, Physiologic endpoints for clinical studies for cystic fibrosis. J Cyst Fibros. $2016 ; 15(4): 416-23$.

25. Calella, P., et al., Association between body composition and pulmonary function in children and young people with cystic fibrosis. Nutrition. 2018; 48: 73-76.

\section{Hosted file}

Tables.docx available at https://authorea.com/users/445988/articles/545342-does-theimplementation-of-children-s-hospital-in-the-home-affect-health-outcomes-for-childrenwith-chronic-respiratory-conditions-a-non-contemporary-retrospective-cohort-comparisonstudy 